\title{
Microsatellite Polymorphism in Japanese Mongrel Dogs
}

\author{
Noboru OISHI ${ }^{1,4)}$, Mari MAEDA ${ }^{1,2)}$, Koichi MAKIMURA ${ }^{2,3)}$, Toshiko SAWAGUCHI ${ }^{5)}$, Makio HAYASHIYA ${ }^{6}$,

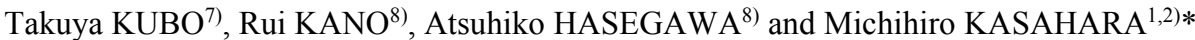 \\ ${ }^{1)}$ Laboratory of Biophysics, School of Medicine, ${ }^{2)}$ Genome Research Center, and ${ }^{3)}$ Institute of Medical Mycology, Teikyo University, \\ Hachioji 192-0395, ${ }^{4}$ Biotechnology Research Center, Teikyo University, Kawasaki 216-0001, 5) Department of Legal Medicine, Tokyo \\ Women's Medical University, Tokyo 162-8666, ${ }^{6}$ Hayashiya Animal Hospital, Uji 611-0002, ${ }^{7}$ Hokuai Animal Hospital, Sapporo 061- \\ 0012 and ${ }^{8)}$ Department of Pathology, School of Veterinary Medicine, Nihon University, Fujisawa 252-8510, Japan
}

(Received 17 February 2005/Accepted 19 June 2005)

ABSTRACT. The genetic variability of 182 unrelated mongrel dogs living in various areas of Japan (from Hokkaido to Okinawa) was studied by collecting their blood. Ten microsatellite loci were chosen from different autosomal chromosomes. After combining a few rare adjoining alleles to allelic classes, it was confirmed that the Hardy-Weinberg equilibrium was attained in each locus. The polymorphic information contents (PICs) of the loci, Ren37A11, Ren48E01, AHTk253, ZuBeCa30, Ren277K09, Ren42N13, AHT130, PEZ03, PEZ12, and $A H T 121$, were $0.58,0.63,0.67,0.67,0.68,0.71,0.79,0.80,0.80$, and 0.80 , and the power of discriminations (PDs) were $0.80,0.85$, $0.87,0.88,0.88,0.89,0.94,0.94,0.94$, and 0.94 , respectively. The combined mean exclusion chance (MEC) was 0.9995 , indicating that these microsatellite loci are useful for kinship testing of Japanese dogs.

KEY WORDS: kinship test, microsatellite, polymorphism.

J. Vet. Med. Sci. 67(10): 1055-1057, 2005

Microsatellites have been used as one of the most useful tools for genetic identification and kinship testing of humans as well as certain animals [12]. Microsatellite analyses were applied to studies of canine pedigrees, $[2,11]$ most notably of American dogs [20]. In regard to the polymorphism of Japanese dogs, blood proteins [25] and mitochondrial DNA [19, 24, 27] have been extensively studied. Recently, several studies utilizing microsatellite markers have been performed $[10,14,17,23,26]$. In these studies, however, evidence of the Hardy-Weinberg equilibrium (HWE) has not been presented. In the current study, we report on the HWE for 10 loci in a Japanese mongrel population.

One hundred and eighty-eight blood samples were collected on FTA Cards (Whatman), which were punched with a $1.2 \mathrm{~mm}$ Harris Micro Punch (Whatman). Materials interfering with PCR were removed from the punched pieces by washing with FTA Purification Reagent (Whatman) 5 times. Ten microsatellite loci were chosen from different autosomal chromosomes. The primer sequences used for each locus were from UniSTS, except for PEZ12 [6], Ren37A11 [13], and $\mathrm{ZuBeCa30}$ [5]. To get clear peaks, oligonucleotide primers for each locus were prepared as Tailed Primer Pairs (Applied Biosystems, 4304979) and labeled with either NED, 6-FAM (6-carboxyfluorescein), or VIC. As a result, all product sizes were reported as having eight base pairs shorter than the observed ones. PCR was performed in a volume of $25 \mu l$ with a punched piece, $0.2 \mathrm{mM}$ dNTP, 0.63 unit of Ex Taq polymerase in Ex Taq buffer (Takara), and a set of primers in various concentrations: AHT130 (NED, 0.1 $\mu \mathrm{M}$ ), AHTk253 (6-FAM, $0.2 \mu \mathrm{M}$ ), Ren37A11 (VIC, 0.2

\footnotetext{
* Correspondence to: Kasahara, M., Laboratory of Biophysics, School of Medicine, Teikyo University, Hachioji, Tokyo 1920395, Japan.
}

$\mu \mathrm{M}$ ), Ren42N13 (6-FAM, $0.1 \mu \mathrm{M}$ ), and Ren277K09 (VIC, $0.2 \mu \mathrm{M})$. The reaction was performed with a GeneAmp PCR System 2400 (Applied Biosystems) for $3 \mathrm{~min}$ at $96^{\circ} \mathrm{C}$, 30 cycles for $30 \mathrm{sec}$ at $96^{\circ} \mathrm{C}, 1 \mathrm{~min}$ at $60^{\circ} \mathrm{C}$, and $30 \mathrm{sec}$ at $72^{\circ} \mathrm{C}$, followed by $5 \mathrm{~min}$ at $72^{\circ} \mathrm{C}$. Another multiplex PCR was performed with a primer set that included AHT121 (6FAM, $0.1 \mu \mathrm{M}$ ), PEZ03 (VIC, $0.1 \mu \mathrm{M}$ ), PEZ12 (NED, 0.1 $\mu \mathrm{M}$ ), Ren48E01 (NED, $0.1 \mu \mathrm{M}$ ), and $Z u B e C a 30$ (6-FAM0.1 $\mu \mathrm{M})$ under the same PCR conditions except for the annealing temperature, which was set at $56^{\circ} \mathrm{C}$. The product size was analyzed by a PRISM 310 Genetic Analyzer with the internal GeneScan 400HD [ROX] size standard (Applied Biosystems). To exclude related samples that affect statistical analyses, the probability of kinship likelihood was calculated between pairs from the same dog owners, on the assumption that the two dogs were siblings from the same parents (The Excel spreadsheet was developed by Aoki [1] and was modified from Essen-Moeller's paternity test [7]). Among these 30 pairs, six showed a probability of kinship likelihood over 0.9. Because these may have been related pairs, one animal from each pair was omitted. The remaining 182 dogs were analyzed as being unrelated. These dogs were from Hokkaido (107), Aomori (2), Tokyo (1), Kanagawa (2), Yamanashi (4), Shizuoka (7), Shiga (1), Kyoto (40), and Okinawa (18).

It is known that rare alleles in a small sample size are prone to disturb statistical estimations [4]. To avoid such complications, a few rare adjoining alleles were grouped to allelic classes to fulfill 2 conditions: 1) that all the expected values of the individual allelic classes are more than 1; and 2) that less than $20 \%$ of the allelic classes exhibit the expected values of less than 5 [4]. The allele frequencies and allelic classes of all the loci are shown in Table 1. For statistical analysis, the observed heterozygosity (Ho: fre- 
quency of heterozygotes), the expected heterozygosity (He: estimated from the allele frequencies)[16], the power of discrimination (PD: probability that two randomly chosen individuals have different phenotypes)[8], the polymorphic information content (PIC: a measure of informativeness related to expected heterozygosity) [3], and the mean exclusion chance (MEC: a priori chance of excluding paternity) [18] of each locus were calculated from the allelic classes.

A chi-square test for the independence between allelic classes was performed for each locus, and the Hardy-Weinberg equilibrium (HWE) was confirmed $(P>0.05)$. Similar results were obtained from Guo and Thompson's exact test for HWE [9] with GENEPOP (version 3.3) [21] and PyPOP [15]. The data obtained with GENEPOP are shown in Table 1. In previous studies of Japanese dogs, however, significant deviations from HWE have been reported [14, 26]. The discrepancies may be due to the fact that this study was based on an unrelated mongrel population while the others were on specific breeds in Japan. In accordance with these observations, our preliminary work on beagles indicated that the proportion of homozygous alleles is greater in beagles than in mongrels.
Although HWE was confirmed in the present mongrel population, it may be possible that a minor difference exists in allelic class distributions among subpopulations because there may be a certain degree of genetic isolation as was observed in the Japanese population [22]. Among the samples from the 3 major sources, Hokkaido, Kyoto, and Okinawa, Fisher's exact tests for population differentiation were performed using GENEPOP. The combined $P$-value of the exact tests was greater than 0.05 , indicating no regional differentiation. By contrast, it was noted from the data based on the blood proteins that the dogs of Hokkaido might be slightly distinct from those of other areas in Japan [25]. It is necessary to collect a larger number of samples from other regions in Japan before reaching a firm conclusion on regional subpopulations.

Given that the combined MEC was calculated to be 0.9995 by using the MEC values shown in Table 1, preliminary kinship tests [1] were performed to see whether the data set obtained in this study is sufficient for practical use. In 2 known pairs of dams and puppies, the probabilities of 0.9999 for parentage likelihood were obtained. In four known pairs of siblings, the probabilities of kinship were

Table 1. Allele frequencies ${ }^{\mathrm{a})}$ and statistical data for 10 microsatellite loci $^{\mathrm{b})}$ in Japanese mongrel dogs

\begin{tabular}{|c|c|c|c|c|c|c|c|c|c|c|}
\hline Locus & Ren $37 A 11$ & Ren48E01 & AHTk253 & $\mathrm{ZuBeCa} 30$ & Ren277K09 & $\operatorname{Ren} 42 N 13$ & AHT130 & PEZ03 & PEZ12 & AHT121 \\
\hline Chromosome & CFA22 & CFA26 & CFA23 & CFA17 & CFA27 & CFA05 & CFA18 & CFA19 & CFA03 & CFA13 \\
\hline Allele 1 & $0.041(247.9)$ & $0.008(175.6)$ & $0.003(275.8)$ & $0.003(217.3)$ & $0.008(202.7)$ & $0.099(148.6)$ & $0.003(97.1)$ & $0.008(104.9)$ & $0.003(251.5)$ & $0.005(90.1)$ \\
\hline Allele 2 & $0.203(252.0)$ & $0.044(186.1)$ & $0.019(277.8)$ & $0.003(219.5)$ & $0.014(204.7)$ & $0.030(156.5)$ & $0.014(99.1)$ & $0.008(107.6)$ & $0.003(264.4)$ & $0.066(92.1)$ \\
\hline Allele 3 & $0.379(256.0)$ & $0.019(188.5)$ & $0.055(279.7)$ & $0.299(221.7)$ & $0.393(206.8)$ & $0.063(158.5)$ & $0.181(101.1)$ & $0.047(111.0)$ & $0.025(267.5)$ & $0.091(94.4)$ \\
\hline Allele 4 & $0.376(258.1)$ & $0.052(190.6)$ & $0.091(281.7)$ & $0.025(223.9)$ & $0.019(208.8)$ & $0.376(160.5)$ & $0.099(103.2)$ & $0.099(113.8)$ & $0.118(271.5)$ & $0.058(96.6)$ \\
\hline Allele 5 & & $0.437(192.7)$ & $0.401(283.6)$ & $0.330(226.0)$ & $0.173(210.9)$ & $0.231(162.5)$ & $0.195(105.3)$ & $0.214(116.8)$ & $0.187(275.6)$ & $0.052(98.9)$ \\
\hline Allele 6 & & $0.308(194.8)$ & $0.203(285.5)$ & $0.181(228.2)$ & $0.085(212.9)$ & $0.159(164.5)$ & $0.102(107.3)$ & $0.228(119.8)$ & $0.181(279.7)$ & $0.170(101.1)$ \\
\hline Allele 7 & & $0.058(196.8)$ & $0.190(287.4)$ & $0.113(230.3)$ & $0.231(215.1)$ & $0.014(166.5)$ & $0.190(109.4)$ & $0.135(122.7)$ & $0.220(283.8)$ & $0.223(103.4)$ \\
\hline Allele 8 & & $0.069(198.8)$ & $0.033(289.3)$ & $0.041(232.5)$ & $0.005(217.1)$ & $0.027(168.6)$ & $0.104(111.5)$ & $0.102(125.7)$ & $0.132(287.8)$ & $0.159(105.6)$ \\
\hline Allele 9 & & $0.005(202.9)$ & $0.005(291.2)$ & $0.005(234.6)$ & $0.069(219.3)$ & & $0.088(113.5)$ & $0.060(128.5)$ & $0.041(291.9)$ & $0.085(107.9)$ \\
\hline Allele 10 & & & & & $0.003(223.6)$ & & $0.025(115.4)$ & $0.055(131.5)$ & $0.016(295.4)$ & $0.052(110.1)$ \\
\hline Allele 11 & & & & & & & & $0.008(134.3)$ & $0.008(299.1)$ & $0.016(112.5)$ \\
\hline Allele 12 & & & & & & & & $0.027(137.3)$ & $0.005(303.1)$ & $0.005(114.5)$ \\
\hline Allele 13 & & & & & & & & $0.008(143.1)$ & $0.019(306.3)$ & $0.005(116.7)$ \\
\hline Allele 14 & & & & & & & & & $0.030(310.4)$ & $0.005(118.8)$ \\
\hline Allele 15 & & & & & & & & & $0.005(314.6)$ & $0.005(120.9)$ \\
\hline Allele 16 & & & & & & & & & $0.003(318.7)$ & \\
\hline Allele 17 & & & & & & & & & $0.003(322.7)$ & \\
\hline Allelic class 1 & $0.245(248-252)$ & $0.124(176-191)$ & $0.168(276-282)$ & $0.330(217-224)$ & $0.415(203-207)$ & $0.099_{(149)}$ & $0.198(97-101)$ & $0.162(105-114)$ & $0.148(252-272)$ & $0.162(90-94)$ \\
\hline Allelic class 2 & 0.379 & 0.437 (193) & $0.401(284)$ & $0.330(226)$ & $0.192(209-211)$ & $0.093(157-159)$ & $0.099_{(103)}$ & $0.214(117)$ & $0.187(276)$ & 0.110 (97-99) \\
\hline Allelic class 3 & 0.376 (258) & 0.308 & 0.203 & 0.181 (228) & $0.085(213)$ & $0.376(161)$ & $0.195(105)$ & $0.228(120)$ & $0.181(280)$ & $0.170(101)$ \\
\hline Allelic class 4 & & $0.132(197-203)$ & $0.228(287-291)$ & $0.159(230-235)$ & $0.231(215-217)$ & $0.231(163)$ & 0.102 (107) & 0.135 & 0.220 & 0.223 \\
\hline Allelic class 5 & & & & & $0.077(219-224)$ & $0.201(165-169)$ & $0.190(109)$ & $0.162(126-129)$ & $0.173(288-292)$ & $0.159(106)$ \\
\hline Allelic class 6 & & & & & & & $0.217(112-115)$ & $0.099(132-143)$ & $0.091(295-323)$ & $0.176(108-121)$ \\
\hline$H o^{\mathrm{c})}$ & 0.634 & & 0.695 & & 0.720 & 0.7 & & & 0.774 & 0.791 \\
\hline $\mathrm{He}$ & 0.655 & 0.682 & 0.718 & 0.724 & 0.725 & 0.746 & 0.820 & 0.822 & 0.824 & 0.827 \\
\hline PD & 0.801 & 0.845 & 0.866 & 0.877 & 0.878 & 0.888 & 0.941 & 0.940 & 0.939 & 0.943 \\
\hline PIC & 0.580 & 0.627 & 0.669 & 0.674 & 0.683 & 0.707 & 0.794 & 0.796 & 0.799 & 0.803 \\
\hline MEC & 0.360 & 0.425 & 0.352 & 0.472 & 0.493 & 0.521 & 0.636 & 0.640 & 0.643 & 0.649 \\
\hline$P($ Chi-square $)$ & 0.827 & 0.189 & 0.653 & 0.334 & 0.091 & 0.725 & 0.276 & 0.492 & 0.077 & 0.204 \\
\hline$P($ Exact $)$ & 0.838 & 0.135 & 0.639 & 0.277 & 0.112 & 0.652 & 0.250 & 0.415 & 0.081 & 0.154 \\
\hline
\end{tabular}

a) Allele frequencies and allelic classes are tabulated. Average allele size in base pairs is shown in parenthesis.

b) Accession numbers for loci are AJ391608 (Ren37A11), AJ391635 (Ren42N13), AJ391660 (Ren48E01), AJ411615 (Ren277K09), AJ269542 (ZuBeCa30), AY536266 (PEZ03) and AY758357 (PEZ12). UniSTS numbers for loci are 262014 (AHT121), 262018 (AHT130), 262080 (AHTk253), 264145 (PEZ03), 264697 (Ren42N13), 264711 (Ren48E01) and 264609 (Ren277K09).

c) Abbreviations used are as follows. Ho: observed heterozygosity, He: expected heterozygosity, PD: power of discrimination, PIC: polymorphic information content, MEC: mean exclusion chance, P(Chi-square): P-value from the chi-square test of the Hardy-Weinberg equilibrium and, $P($ Exact $)$ : P-value from the exact test of the Hardy-Weinberg equilibrium calculated using GENEPOP [21]. 
over 0.98. These results indicated that the present data set is useful for kinship analysis of Japanese dogs.

ACKNOWLEDGEMENT. We would like to thank Dr. A. Akane (Kansai Medical University) for his critical reading of this manuscript.

\section{REFERENCES}

1. Aoki, Y., Hashiyada, M., Morioka, A., Nata, M. and Sagisaka, K. 1997. Jpn. J. Legal Med. 51: 196-204 (in Japanese).

2. Binns, M.M., Holmes, N.G., Marti, E. and Bowen, N. 1995. J. Small Anim. Pract. 36: 493-497.

3. Botstein, D., White, R.L., Skolnick, M. and Davis, R.W. 1980. Am. J. Hum. Genet. 32: 314-331.

4. Cochran, W.G. 1954. Biometrics 110: 417-451.

5. Dolf, G., Schelling, C., Stahlberger-Saitbekova, N., Fu, B., Schlapfer, J. and Yang, F. 2000. Anim. Genet. 31: 411-412.

6. Eichmann, C., Berger, B. and Parson, W. 2004. Int. J. Legal Med. 118: 249-266.

7. Essen-Moeller, E. 1938. Mitt. Anthrop. Ges. Wien 68: 9-53.

8. Grunbaum, B.W., Selvin, S., Pace, N. and Black, D.M. 1978. J. Forensic Sci. 23: 577-587.

9. Guo, S.W. and Thompson, E.A. 1992. Biometrics 48: 361-372.

10. Ichikawa, Y., Takagi, K., Tsumagari, S., Ishihama, K., Morita, M., Kanemaki, M., Takeishi, M. and Takahashi, H. 2001. J. Vet. Med. Sci. 63: 1209-1213.

11. Irion, D.N., Schaffer, A.L., Famula, T.R., Eggleston, M.L., Hughes, S.S. and Pedersen, N.C. 2003. J. Hered. 94: 81-87.

12. Jones, A. G. and Ardren, W. R. 2003. Mol. Ecol. 12: 25112523.

13. Jouquand, S., Priat, C., Hitte, C., Lachaume, P., Andre, C. and
Galibert, F. 2000. Anim. Genet. 31: 266-272.

14. Kim, K.S., Tanabe, Y., Park, C.K. and Ha, J.H. 2001. J. Hered. 92: 398-403.

15. Lancaster, A., Nelson, M.P., Single, R.M., Meyer, D. and Thomson, G. 2003. pp. 514-525. In: Pacific Symposium on Biocomputing 8 (Altman, R.B., Dunker, A.K., Hunter, L. and Klein, T.E. eds.), World Scientific, Singapore.

16. Nei, M. and Roychoudhury, A.K. 1974. Genetics 76: 379-390.

17. Niimi, Y., Inoue-Murayama, M., Murayama, Y., Ito, S. and Iwasaki, T. 1999. J. Vet. Med. Sci. 61: 1281-1286.

18. Ohno, Y., Sebetan, I.M. and Akaishi, S. 1982. Forensic Sci. Int. 19: 93-98.

19. Okumura, N., Ishiguro, N., Nakano, M., Matsui, A. and Sahara, M. 1996. Anim. Genet. 27: 397-405.

20. Parker, H.G., Kim, L.V., Sutter, N.B., Carlson, S., Lorentzen, T.D., Malek, T.B., Johnson, G.S., DeFrance, H.B., Ostrander, E.A. and Kruglyak, L. 2004. Science 304: 1160-1164.

21. Raymond, M. and Rousset, F. 1995. J. Hered. 86: 248-249.

22. Tajima, A., Hayami, M., Tokunaga K., Juji, T., Matsuo, M., Marzuki, S., Omoto, K. and Horai, S. 2004. J. Hum. Genet. 49: 187-193.

23. Takagi, K., Tsumagari, S., Ichikawa, Y., Ishihama, K., Morita, M., Kanemaki, M. and Takeishi, M. 2001. Cell Mol. Biol. (Noisy-le-grand) 47: OL181-OL185.

24. Takahasi, S., Miyahara, K., Ishikawa, H., Ishiguro, N. and Suzuki, M. 2002. J. Vet. Med. Sci. 64: 255-259.

25. Tanabe, Y. 1990. Prog. Clin. Biol. Res. 344: 619-637.

26. Tsuchida, S., Ikemoto, S. and Tagawa, M. 2001. J. Vet. Med. Sci. 63: 479-481.

27. Tsuda, K., Kikkawa, Y., Yonekawa, H. and Tanabe, Y. 1997. Genes Genet. Syst. 72: 229-238. 